\title{
Advanced Numerical Modeling in Engineering - FDEM: A Micro- Mechanical Appproach that Bridges Across Scales
}

\author{
Giovanni Grasselli \\ University of Toronto, Canada
}

The hybrid finite/discrete element model (FDEM) is an innovative numerical technology that combines the advantages of large-strain finite elements with those of the discrete elements, allowing for explicitly modelling the propagation of cracks into heterogeneous brittle materials, as well as the interaction between their different constituent phases and materials. By modelling these physical interactions at the micro-scale, FDEM is able to reproduce the response of the tested material at multiple scales as an emergent property of the model. Clearly, as any other numerical approach, the reliability of a FDEM simulation is highly dependent on the used input material properties. A micro-characterization of the studies material, together with advanced imaging performed inside a micro x-ray computed tomography machine (microCT), are currently adopted to inform and constrain the FDEM model. Following an overview of the FDEM basic principles, three engineering applications will be presented and discussed within the keynote lecture. 